\title{
Phason Dispersion and Magnetic-Field-Induced Band Gap in a Ferroelectric Liquid Crystal
}

\author{
I. Muševič, B. Žekš, and R. Blinc \\ J. Stefan Institute, Jamova 39, 61000 Ljubljana, Slovenia \\ H. A. Wierenga and Th. Rasing \\ Research Institute for Materials and High Field Magnet Laboratory, University of Nijmegen, \\ NL-6525 ED Nijmegen, The Netherlands \\ (Received 30 December 1991)
}

\begin{abstract}
The phason dispersion and the dielectric response of a ferroelectric $\mathrm{Sm}-\mathrm{C}^{*}$ liquid crystal in an external magnetic field have been measured. A magnetic-field-induced splitting of the phason branch into an acousticlike and an opticlike mode was observed, indicating a crossover between the plane-wave-like and solitonlike phason dynamics. The resulting band gap at $q=0$ is a direct consequence of the broken continuous helicoidal symmetry of the $\mathrm{Sm}-C^{*}$ phase in a transverse magnetic field.
\end{abstract}

PACS numbers: 61.30.Gd, 64.70.Md, 64.70.Rh, 78.35.+c

In the Sm- $C^{*}$ phase of a ferroelectric liquid crystal, rodlike molecules with a transverse dipole moment are arranged in liquidlike smectic layers. The director $\mathbf{n}(\mathbf{r})$ that describes the time-averaged orientation of the long molecular axis is tilted with respect to the layer normal at an angle $\theta$ and precesses with a period $p_{0}$ on moving from one layer to another. Usually $p_{0}$ is much larger than the interlayer distance so that the system can be considered as a helicoidal continuum. The symmetry of each smectic layer is $C_{2}$.

In the long-wavelength limit the spectrum of elementary excitations consists of two branches [1,2]. The amplitudon branch represents the variation in the magnitude of the tilt angle and is observable only close to the Sm$C^{*} \rightarrow \mathrm{Sm}-A$ transition. The phason branch represents a collective plane-wave-like excitation of the phase $\Phi(\mathbf{r}, t)$ of the director field. Because of the continuous helicoidal symmetry, the phason excitation displays a plane-wave behavior with a parabolic dispersion relation.

A static magnetic field [3] $\mathbf{H}=(0, H, 0)$ perpendicular to the helical axis $\mathbf{e}_{z}$ tends to align the molecules in the direction of the field if the anisotropy of the magnetic susceptibility $\Delta \chi=\chi_{\|}-\chi_{\perp}$ is positive. The helical structure is distorted in a solitonlike manner and this distortion is accompanied by an increase of the period $p(H)$ of the $\mathrm{Sm}-\mathrm{C}^{*}$ phase. The solitonlike distortion breaks the continuous helicoidal symmetry of the unperturbed Sm$C^{*}$ phase. The remaining symmetry element is a discrete one, i.e., a translation over a distance $p / 2$ along the helix, followed by a rotation through an angle $\pi$ around the helix. High magnetic fields completely unwind the helical structure and the $\mathrm{Sm}-\mathrm{C}^{*}$ phase transforms into a uniform Sm-C phase at the critical field $H_{c}$.

The breaking of the continuous helicoidal symmetry has some fascinating implications for the nature of phason dynamics. Phason eigenfunctions should obtain the Bloch form and the concept of a Brillouin zone (BZ) has to be introduced, reflecting the appearance of a periodic potential for phason propagation. This should result in a splitting of the phason branch into an acoustic- like and an opticlike branch, separated by a gap. The magnetic field has here a similar role as the "lock-in" lattice term in incommensurate solids. The difference is that here the magnetic field can be continuously varied and thus the "soliton" regime and the magnitude of the periodic potential can be externally controlled at any given temperature which is not the case in incommensurate systems.

In this Letter, we report what we believe to be the first experimental observation of the splitting of the phason branch into an acousticlike and an opticlike mode. The experiment was performed in the $\mathrm{Sm}-\mathrm{C}^{*}$ phase of a ferroelectric liquid crystal and the splitting was induced by an external transverse magnetic field. The phason dispersion curves were determined by quasielastic light scattering spectroscopy, whereas the magnetic-field-induced band gap was measured by both light scattering and dielectric relaxation spectroscopy. The splitting between the two branches was observed even for very small fields, where the change of the period of the helix is too small to be detected.

The dynamics of the phase fluctuations $[1,2,4-6]$, propagating along the helical axis of a distorted $\mathrm{Sm}-\mathrm{C}^{*}$ phase, can be derived from the Landau thermodynamic potential $[7,8]$. Expressing the $\mathrm{Sm}-C^{*}$ order parameter $\xi$ as

$$
\xi_{1}(z, t)=\theta \cos \Phi(z, t), \quad \xi_{2}(z, t)=\theta \sin \Phi(z, t),
$$

one obtains in the constant amplitude approximation [3] the phase-dependent part of the nonequilibrium freeenergy density as

$g(\Phi)=-\Lambda \theta^{2} \frac{\partial \Phi}{\partial z}+\frac{1}{2} K_{33} \theta^{2}\left(\frac{\partial \Phi}{\partial z}\right)^{2}-\frac{1}{2} \Delta \chi H^{2} \theta^{2} \sin ^{2} \Phi$.

Here $\Lambda$ is the Lifshitz term, $K_{33}$ is the torsional elastic constant, and $\Delta \chi$ is the diamagnetic anisotropy. After minimizing $g(\Phi)$ with respect to $\Phi(z)$, one obtains the static solution $\Phi_{0}(z)$ of the sine-Gordon equation in the 
well-known form of a $\pi$-soliton lattice:

$$
\sin \Phi_{0}(z)=\operatorname{sn}(u, k) .
$$

Here $u=z / \xi k$ is the argument of a Jacobian elliptic function of modulus $k$, and $\xi$ is the magnetic coherence length, $\xi^{2}=K_{33} / \Delta \chi H^{2}$. The modulus $k$ is determined by the external field and satisfies the equations $k=(H /$ $\left.H_{c}\right) E(k)$ and $H_{c}=\left(\pi^{2} / p_{0}\right)\left(K_{33} / \Delta \chi\right)^{1 / 2}$. Here $E(k)$ is the complete elliptic integral of the second kind. By taking $\Phi(z, t)=\Phi_{0}(z)+\delta \Phi(z, t)$, where $\delta \Phi(z, t)$ $=\Psi(z) e^{-t / \tau(H)}$, one obtains after applying the LandauKhalatnikov equations of motion, linearized in $\Psi(z)$, Lamé's equation of order one for the eigenvectors of the phase fluctuations amplitude [4-6] $\Psi(u)$ :

$$
\frac{d^{2} \Psi}{d u^{2}}+\left[h-2 k^{2} \operatorname{sn}^{2}(u, k)\right] \Psi=0 .
$$

Here $h=k^{2}\left\{\xi^{2} \gamma /\left[\tau(H) K_{33}\right]+1\right\}$ and $\gamma$ is the viscosity of the $\mathrm{Sm}-C^{*}$ phase. General solutions of Lamé's equation (4) in the form of the Bloch waves are known $[6,9,10]$, and the corresponding wave vector $\kappa$ can be ascribed on the basis of symmetry transformation properties of these eigenfunctions. It should be stressed that in the experiment one observes the excitations of the order parameter $\xi$, whereas Eq. (4) describes the excitations of the phase $\delta \Phi_{\kappa}(z, t)$. It can be shown from Eqs. (1) and (3) that the phase excitation $\delta \Phi_{\kappa}$ with a wave vector $\kappa$ and the relaxation rate $\tau^{-1}$ is observable as an excitation of the order parameter $\xi$ at the wave vectors $q=\kappa \pm q_{c}$, where $q_{c}(H)=2 \pi / p(H)$. This has the consequence that the center of the phason BZ (i.e., $\kappa=0$, see below), introduced by the periodic potential $k^{2} \operatorname{sn}^{2}(u, k)$ in Eq. (4), is observable near the first Bragg peak at $q= \pm q_{c}$ in the experiment.

The eigenfunctions of particular interest are those with the wave vectors $q=0$ and $q= \pm q_{c}(H)$, corresponding to the edges and to the center of the BZ. These eigenfunctions $\Psi^{(i)}$ and their corresponding eigenvalues $\tau^{-1}$ are

$$
\begin{aligned}
& \Psi^{(1)}=\operatorname{dn}(u, k), \quad \tau{ }^{-1}\left(q= \pm q_{c}(H)\right)=0, \\
& \Psi^{(2)}=\operatorname{cn}(u, k), \quad \tau=^{-1}(q=0)=\frac{1-k^{2}}{k^{2}} \frac{\Delta \chi}{\gamma} H^{2}, \\
& \Psi^{(3)}=\operatorname{sn}(u, k), \quad \tau_{+}^{-1}(q=0)=\frac{1}{k^{2}} \frac{\Delta \chi}{\gamma} H^{2} .
\end{aligned}
$$

Here $\operatorname{dn}(u, k), \operatorname{cn}(u, k)$, and $\operatorname{sn}(u, k)$ are the Jacobian delta, cosine, and sine amplitudes, respectively. At $H \rightarrow 0, k \rightarrow 0$, so that $\tau^{-1}(q=0)=\tau_{+}^{-1}(q=0)$ $=\left(K_{3} / \gamma\right) q_{c}^{2}$.

What is the physical interpretation of these solutions? $\Psi^{(1)}$ is a Goldstone mode, recovering the broken continuous $\left(D_{\infty}\right)$ symmetry of the $\operatorname{Sm}-A$ phase. For $H=0$ this mode amounts to a uniform rotation of the whole helix, or, what is the same, to a sliding of the helix. For $H \neq 0$ this mode represents a uniform translation of the soliton lattice as a whole, resulting in a gapless mode at $q= \pm q_{c}$. The solution $\Psi^{(2)}=\operatorname{cn}(u, k)$ represents an acousticlike phase excitation that has the nodes in the middle of the commensurate regions between the solitonlike domain walls. Thus, $\Psi^{(2)}$ shifts the centers of these "domain walls." The relaxation rate of this mode decreases with increasing magnetic field. The solution $\Psi^{(3)}=\mathrm{sn}(u, k)$ represents an opticlike phase excitation that changes the width of the domain walls. The corresponding relaxation rate increases with increasing magnetic field.

As a result of quadratic diamagnetic coupling, the phason excitation sees in the presence of $H \neq 0$ a periodic potential $k^{2} \mathrm{sn}^{2}(u, k)$ with periodicity $p / 2$. This symmetry breaking introduces a Brillouin zone defined by $-2 \pi / p \leq \kappa \leq 2 \pi / p$ and implies a Bloch form of the phason eigenfunctions. The coupling between the phason modes at $\kappa= \pm q_{c}$ causes a gap $G(H)$ to appear at the edges of the $\mathrm{BZ}$, separating the acousticlike mode $\Psi^{(2)}$ from the opticlike mode $\Psi^{(3)}$. In the experiment, the phason band gap $G(H)$ is observable at $q=0$ as shown in Fig. 1:

$G(H, q=0)=\tau_{+}^{-1}(q=0)-\tau-^{-1}(q=0)=(\Delta \chi / \gamma) H^{2}$.

As shown by Sutherland [10], in such a system there is only one gap.

In the $\mathrm{Sm}-\mathrm{C}^{*}$ phase, the fluctuations of the phase $\Phi(z, t)$ of the order parameter induce the fluctuations of the spontaneous dipole moment $\mathbf{P}(\mathbf{r}, t)$ and the local dielectric tensor $\epsilon(\mathbf{r}, t)$. Whereas quasielastic light scattering spectroscopy allows for the measurement of the phason dispersion [1,2], dielectric spectroscopy will give information on the phason dynamics at $q=0$. It can be shown from Eqs. (6) and (7) that the acousticlike mode $\Psi^{(2)}$ represents a fluctuation of the space-averaged spontaneous electric polarization $\delta \mathbf{P}=(\delta P, 0,0)$, which is perpendicular to the magnetic field $H$. In a similar way, the opticlike phason $\Psi^{(3)}$ represents a fluctuation of spaceaveraged polarization $\delta \mathrm{P}=(0, \delta P, 0)$ in the direction of the magnetic field.

The experiment was performed in a mixture consisting

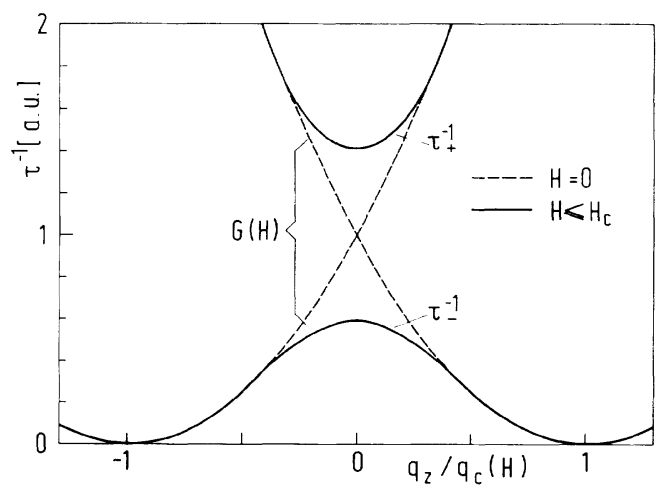

FIG. 1. The effect of an external magnetic field on the phason dispersion in the $\mathrm{Sm}-\mathrm{C}^{*}$ phase of a ferroelectric liquid crystal. 


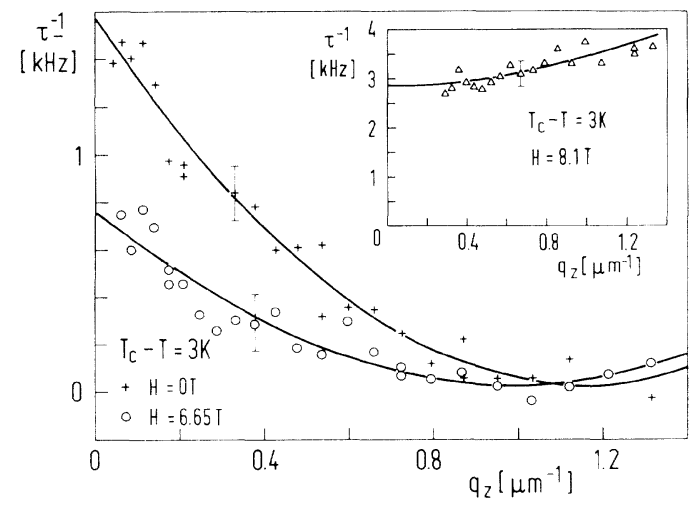

FIG. 2. Dispersion of the acousticlike phason branch as determined at different magnetic fields. Solid lines represent the best parabolic fits $\tau^{-1}=\left(K_{3} / \gamma\right)\left(q-q_{c}\right)^{2}$ : At $H=0$ we get $K_{33} / \gamma=1.1 \times 10^{3} \mu \mathrm{m}^{2} \mathrm{~s}^{-1}$ and $p_{0}=5.7 \mu \mathrm{m}$, whereas at $H=6.65$ $\mathrm{T}$ we get $K_{33} / \gamma=0.76 \times 10^{3} \mu \mathrm{m}^{2} \mathrm{~s}^{-1}$ and $p=6.2 \mu \mathrm{m}$. Inset: The phason dispersion at $8.1 \mathrm{~T}$ with $K_{3} / \gamma=0.6 \times 10^{3} \mu \mathrm{m}^{2} \mathrm{~s}^{-1}$ and $q_{c}=0$.

of $35 \%$ of pure chiral liquid crystal $4-\left(2^{\prime}\right.$-methylbutyl)phenyl $4^{\prime}$-n-octylbiphenyl-4-carboxylate (CE-8) and $65 \%$ of racemic liquid crystal CE-8R. The phason dispersion curves were measured with a miniature quasielastic light scattering spectrometer, fitting into a $60-\mathrm{mm}$ Bitter magnet. A He-Ne ordinary polarized laser beam was slightly focused to a $50-\mu \mathrm{m}$ spot, illuminating a $120-\mu \mathrm{m}$-thick, homeotropically aligned liquid-crystal sample, placed in a thermostated oven $( \pm 0.1 \mathrm{~K})$ in the center of the magnet. The scattered extraordinary light was collected into a multimode fiber and fed into a photomultiplier, placed far away from the magnet. The axis of the collecting optics was positioned at an angle $1^{\circ}$ from the incoming light direction, thus assuring a heterodyne detection of the autocorrelation function of the scattered light intensity. In addition, the field dependence of the period of the helix as well as the dielectic response was determined. The dielectric response was measured with $\mathbf{E} \| \mathbf{H} \perp \mathbf{q}_{c}(\mathbf{H})$, thus probing the opticlike phason mode at $q=0$. For the experimental details, see Ref. [11].

The phason dispersions were measured $3 \mathrm{~K}$ below $T_{c}$ at several magnetic fields. The magnetic field was swept slowly to the desired value, and then the system was left for $30 \mathrm{~min}$ to reach the equilibrium state. Phason dispersions as measured at $0,6.65$, and $8.1 \mathrm{~T}$ are shown in Fig. 2. One can clearly observe the change in the shape of the phason dispersion curves, being most pronounced at $q=0$. The best parabolic fit to the zero-field dispersion gives the value $K_{33} / \gamma=1.1 \times 10^{3} \mu \mathrm{m}^{2} \mathrm{~s}^{-1}$ and $p_{0}=5.7 \mu \mathrm{m}$. A parabolic fit of the phason dispersions at 6.65 and $8.1 \mathrm{~T}$ shows a systematic decrease of the constant $K_{33} / \gamma$, together with a shift of the center of the dispersion, corresponding to a change of the helical pitch. The minimum of the phason dispersion at $8.1 \mathrm{~T}$ is shifted to $q=0$, clearly indicating the appearance of the homogeneous $\mathrm{Sm}-\mathrm{C}$ phase. On the basis of these observations, we conclude

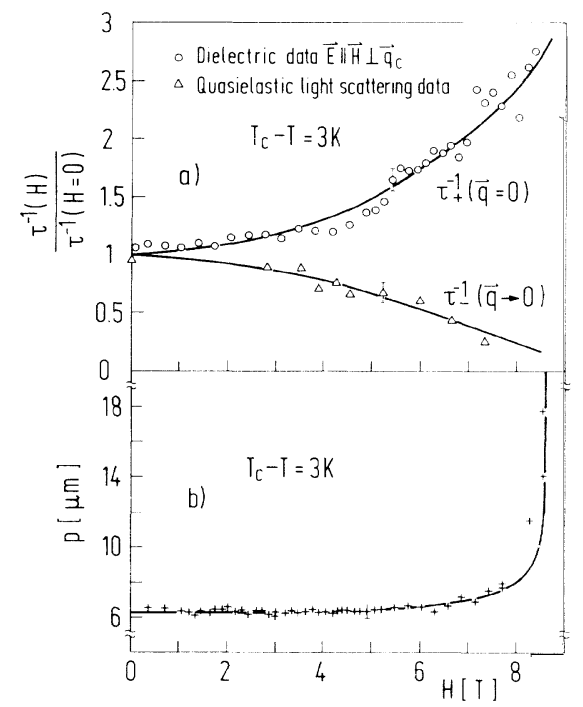

FIG. 3. Magnetic-field dependence of the (a) normalized relaxation rates of the optic and acoustic phason modes at $q=0$ and of the (b) period of the helix. Solid lines in (a) represent the best fits by Eqs. (6) and (7) with $\Delta \chi / \gamma=48 \mathrm{~T}^{-2} \mathrm{~s}^{-1}$ and $H_{c}=8.1 \mathrm{~T}$ for the optic phason mode $\tau{ }^{-1}(q=0)$ and $\Delta \chi / \gamma=42$ $\mathrm{T}^{-2} \mathrm{~s}^{-1}$ and $H_{c}=9.7 \mathrm{~T}$ for the acoustic phason mode $\tau=-1(q=0)$. The solid line in (b) represents the constant amplitude approximation fit $[3,5]$ with $p_{0}=6.2 \mu \mathrm{m}$ and $H_{c}=8.6 \mathrm{~T}$.

that in the quasielastic light scattering experiment below $H_{c}$ we observe the dominant acousticlike phason branch. The intensity of the higher-frequency opticlike phason branch is covered by the high intensity signal of the acoustic branch and is observable only as a weak signal above $H_{c}$, where the acoustic branch disappears.

Figure 3(a) shows the magnetic-field dependence of the normalized phason relaxation rates at $q=0$, as obtained from quasielastic light scattering and dielectric response. One can clearly observe a large decrease of the relaxation rates of the acousticlike phason mode at fields far below the critical field. At the same time, we can observe a strong increase of the relaxation rates of the opticlike phason mode, as determined from the dielectric response. Figure 3(b) shows the magnetic-field dependence of the period of the helix, measured at the same temperature. By comparing the magnetic-field dependences of the two phason modes to the magnetic-field dependence of the period of the helix, one sees a striking difference. Whereas one can hardly see any change in the period up to $6 \mathrm{~T}$, the magnitude of the gap separating the optic and the acoustic phason branches is of the order of the zerofield phason relaxation rate, i.e., $1.5 \mathrm{kHz}$. This is a clear indication of a crossover behavior of the phason dynamics between the plane-wave dynamics in the unperturbed helicoidal structure and the solitonlike dynamics in a distorted helicoidal $\mathrm{Sm}-C^{*}$ structure.

In Fig. 4 we present the magnetic-field dependence of the gap $G(H, q=0)$ as determined from optic and dielec- 


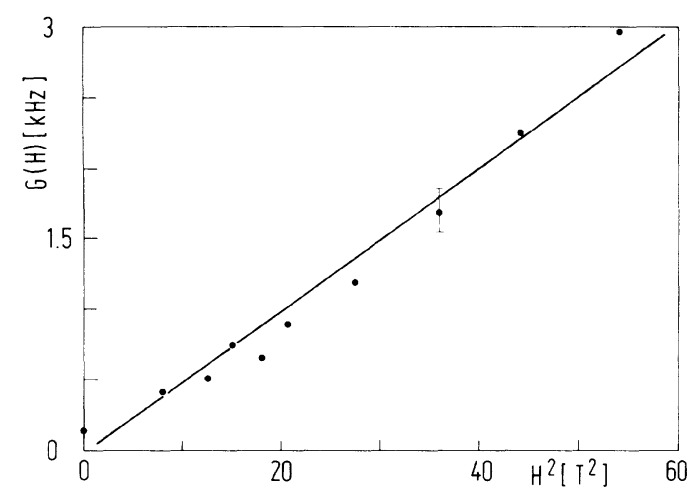

FIG. 4. Magnetic-field dependence of the gap $G(H)$, separating the optic and acoustic phason modes at $q=0$, $T_{c}-T=3 \mathrm{~K}$. The difference between the normalized optic and acoustic phason relaxation rates was multiplied by the mean value of $1.5 \mathrm{kHz}$ for both relaxation rates at zero field (see text) to obtain $G(H)$.

tric measurements. The gap is proportional to the square of the magnetic field. From the least-mean-squares fit following Eq. (8), we determined the value $\Delta \chi / \gamma=50$ $\mathrm{T}^{-2} \mathrm{~S}^{-1}$ for the data shown in Fig. 4. This is in good agreement with the values $\Delta \chi / \gamma=42 \quad \mathrm{~T}^{-2} \mathrm{~s}^{-1}$ and $\Delta \chi / \gamma=48 \mathrm{~T}^{-2} \mathrm{~s}^{-1}$ as obtained by fitting the acoustic and optic phason branches with Eqs. (6) and (7), respectively. The agreement of the critical magnetic fields, as obtained by this fitting is worse, since we obtain the value $H_{c}=8.1$ $T$ for the optic branch and $H_{c}=9.7 \mathrm{~T}$ for the acoustic branch. This difference can be explained by the fact that the measurements were done on different samples with slightly different periods of the helix. This results in a scattering in the zero-field relaxation rates, where we obtain a zero-field relaxation rate of $1.6 \pm 0.2 \mathrm{kHz}$ for the acoustic branch and $1.4 \pm 0.2 \mathrm{kHz}$ for the optic phason branch. This scattering, however, does not significantly influence the physical interpretation of the observed effects.
In conclusion, we have observed for the first time the magnetically induced splitting of the phason branch in the $\mathrm{Sm}-\mathrm{C}^{*}$ phase of a ferroelectric liquid crystal into an acousticlike and an opticlike mode. The appearance of a single gap in the phason excitation spectrum is here connected with the crossover from the plane wave to the soliton lattice modulation regime, and is the result of the breaking of the original continuous symmetry by the external field. We believe that the above observations and conclusions may lead to a better understanding of the dynamical properties of modulated structures in external fields and incommensurate systems in general.

We would like to thank Professor M. Copič for helpful discussions, Henk Hesselman for his kind assistance during our experiments in Nijmegen, and $\mathbf{J}$. Dijkhuis for kindly lending us the autocorrelator.

[1] I. Muševič, R. Blinc, B. Žekš, C. Filipič, M. Čopič, A. Seppen, P. Wyder, and A. Levanyuk, Phys. Rev. Lett. 60, 1530 (1988).

[2] I. Drevenšek, I. Muševič, and M. Čopič, Phys. Rev. A 41, 923 (1990).

[3] I. Mušcvič, B. Žeks, R. Blinc, Th. Rasing, and P. Wyder, Phys. Rev. Lett. 48, 192 (1982); R. Blinc, I. Muševič, and B. Žekš, Phys. Scr. T35, 38 (1991).

[4] C. Fan, L. Kramer, and M. J. Stephen, Phys. Rev. A 2, 2482 (1970).

[5] J. D. Parsons and C. F. Hayes, Phys. Rev. A 9, 2652 (1974).

[6] M. Yamashita, H. Kimura, and H. Nakano, Prog. Theor. Phys. 65, 1504 (1981).

[7] S. A. Pikin and V. L. Indenbom, Usp. Fiz. Nauk 125, 251 (1978) [Sov. Phys. Usp. 21, 487 (1978)].

[8] R. Blinc and B. Žekš, Phys. Rev. A 18, 740 (1978).

[9] E. T. Whittaker and T. G. N. Watson, "Modern Analysis" (Cambridge Univ. Press, Cambridge, England, to be published).

[10] B. Sutherland, Phys. Rev. A 8, 2514 (1973).

[11] I. Muševič, B. Žekš, R. Blinc, H. A. Wierenga, and Th. Rasing, Physica (Amsterdam) (to be published). 